\title{
Meta
}

Journal des traducteurs

Translators' Journal

\section{Experts versus novices : l'utilisation de sources d'information pendant le processus de traduction}

\section{Alexander Künzli}

Volume 46, numéro 3, septembre 2001

URI : https://id.erudit.org/iderudit/003363ar

DOI : https://doi.org/10.7202/003363ar

Aller au sommaire du numéro

Éditeur(s)

Les Presses de l'Université de Montréal

ISSN

0026-0452 (imprimé)

1492-1421 (numérique)

Découvrir la revue

Citer cet article

Künzli, A. (2001). Experts versus novices : l'utilisation de sources d'information pendant le processus de traduction. Meta, 46(3), 507-523.

https://doi.org/10.7202/003363ar

\section{Résumé de l'article}

Cette étude a pour objectif d'examiner l'utilisation des sources d'information au cours du processus de traduction. Trois traducteurs professionnels et trois étudiants en traduction avaient pour tâche de traduire un texte en pensant à voix haute. Les résultats révèlent une corrélation entre le nombre de sources d'information consultées, l'expérience de la traduction et la qualité de la traduction. En revanche, la qualité de la traduction n'est pas liée à la préférence pour un certain type de source d'information (par exemple, dictionnaire monolingue vs dictionnaire bilingue). Notre étude a des implications de deux ordres. Sur le plan méthodologique, il convient de rappeler que des résultats corrélationnels ne permettent pas d'établir des liens de cause à effet. Sur le plan de la pédagogie de la traduction, nos données mettent en cause la critique souvent avancée à l'égard de l'usage de dictionnaires bilingues. 


\title{
Experts versus novices: \\ l'utilisation de sources d'information pendant le processus de traduction'
}

\author{
ALEXANDER KÜNZLI \\ Université de Stockholm, Stockholm, Suède
}

\section{RÉSUMÉ}

Cette étude a pour objectif d'examiner l'utilisation des sources d'information au cours du processus de traduction. Trois traducteurs professionnels et trois étudiants en traduction avaient pour tâche de traduire un texte en pensant à voix haute. Les résultats révèlent une corrélation entre le nombre de sources d'information consultées, l'expérience de la traduction et la qualité de la traduction. En revanche, la qualité de la traduction n'est pas liée à la préférence pour un certain type de source d'information (par exemple, dictionnaire monolingue vs dictionnaire bilingue). Notre étude a des implications de deux ordres. Sur le plan méthodologique, il convient de rappeler que des résultats corrélationnels ne permettent pas d'établir des liens de cause à effet. Sur le plan de la pédagogie de la traduction, nos données mettent en cause la critique souvent avancée à l'égard de l'usage de dictionnaires bilingues.

\begin{abstract}
This study investigates the use of information sources during the translation process. Three professional translators and three translation students were asked to translate a short text while thinking aloud. The results show a correlation between the range of information sources used, expertise of translation and translation quality. However, the preference of a certain type of source (e.g., monolingal vs. bilingual dictionaries) is not associated with translation quality. The results have implications for translation research methodology, as it should be reminded that correlational results do not permit the attribution of causal links, and translation pedagogy, where the criticism often expressed towards the use of bilingual dictionaries seems unjustified in the light of our data.
\end{abstract}

\section{MOTS-CLÉS/KEYWORDS}

dictionnaires, pédagogie de la traduction, processus de traduction, qualité de la traduction, sources d'information

\section{Cadre épistémologique de référence}

En traductologie, l'usage des sources d'information par les étudiants en traduction et les traducteurs professionnels mérite une attention particulière. Si la question de l'utilisation des sources d'information est assez bien documentée dans des approches normatives ou pédagogiques de la traduction (par exemple, Gile, 1995; Schäffner, 1998; Snell-Hornby, 1995), peu de travaux empiriques rendent compte de l'utilisation réelle de ces sources par les traducteurs. L'objectif de notre étude étant de contribuer à combler cette lacune, nous allons d'abord donner un aperçu des travaux qui se rapportent à ce sujet.

En 1986, Krings a donné le coup d'envoi à la recherche empirique sur les processus de traduction à l'aide de protocoles de réflexion parlée, en consacrant une partie 
de son œuvre à l'étude des stratégies de consultation de dictionnaires chez des sujets traduisant des textes du français vers l'allemand et vice versa. Il constate que (a) le dictionnaire bilingue est de loin l'outil le plus consulté; (b) le dictionnaire monolingue ne joue qu'un rôle marginal et est consulté uniquement en cas de consultation improductive du dictionnaire bilingue; (c) d'autres sources ne sont pas consultées. De plus, Krings (1986) montre que l'usage du dictionnaire bilingue conduit dans 2 cas sur 3 à une solution correcte du problème à l'origine de la consultation et que ce taux aurait dépassé les deux tiers si les sujets avaient traité les informations contenues dans le dictionnaire bilingue de façon optimale.

Jääskeläinen (1989) examine l'utilisation de sources d'information en transférant le cadre d'observation de Krings (1986) à deux groupes divisés selon leur expérience de la traduction: un groupe d'étudiants débutants en traduction et un groupe d'étudiants avancés. Elle observe que (a) de manière générale, les étudiants débutants ont plus souvent recours à des sources d'information que les étudiants avancés; (b) les étudiants débutants utilisent plus de dictionnaires bilingues que d'autres sources, alors que les étudiants avancés préfèrent travailler avec des dictionnaires monolingues et d'autres sources d'information monolingues (encyclopédies, etc.); (c) les étudiants débutants se tournent en général vers des dictionnaires bilingues avant toute autre source pour résoudre un problème ( first look-ups»), alors que, dans la majorité des cas, les étudiants avancés commencent leurs recherches par le recours à des dictionnaires monolingues. Jääskeläinen (1989) relève néanmoins que sur le plan quantitatif, les différences entre les deux groupes sont faibles. Elles sont plutôt d'ordre qualitatif et concernent la façon dont les deux groupes utilisent les différentes sources d'information. Quant à la qualité des traductions, l'auteur dit observer une corrélation entre la qualité de la traduction et l'utilisation de dictionnaires. En effet, les traductions jugées les meilleures sont celles des étudiants avancés qui font le plus appel à des dictionnaires monolingues.

Dans son ouvrage paru en 1995, Kussmaul consacre lui aussi un chapitre à l'étude de l'utilisation des sources d'information chez des étudiants en traduction. Se plaçant dans la position de l'enseignant en traduction, l'auteur préconise une approche critique vis-à-vis des dictionnaires bilingues - peut-être trop critique à la lumière des résultats de Krings (1986)? — et en déconseille même l'usage. Ainsi, il affirme que ses études sur les processus de traduction, faites grâce aux protocoles de réflexion parlée, ont révélé que les étudiants s'appuient trop sur des dictionnaires bilingues. Nous aurions néanmoins souhaité un nombre plus élevé d'extraits de protocoles permettant d'attester ce phénomène.

Englund Dimitrova \& Jonasson (à paraître) ont également étudié l'utilisation de dictionnaires chez des étudiants en traduction et des traducteurs professionnels, et montrent qu'il n'y a pas de différences entre les deux groupes pour la fréquence totale d'utilisation de dictionnaires (traducteurs professionnels vs étudiants en traduction). Dans l'analyse des types de dictionnaires utilisés, les auteurs constatent néanmoins que les traducteurs professionnels ont davantage recours à des dictionnaires monolingues que bilingues. Le comportement du groupe des étudiants, en revanche, n'est pas homogène. À l'instar de Jääskeläinen (1989), les auteurs affirment observer une corrélation entre l'utilisation de certains types de dictionnaires et la qualité de la traduction: les traductions jugées les meilleures sont celles des deux traducteurs professionnels qui préfèrent l'usage de dictionnaires monolingues, les 
traductions des deux étudiants préférant consulter des dictionnaires monolingues sont qualifiées de moyennes; enfin les traductions des deux étudiants qui utilisent avant tout des dictionnaires bilingues sont considérées comme inacceptables. Les auteurs en tirent la conclusion, à l'instar de Kussmaul (1995), que c'est l'utilisation irraisonnée du dictionnaire bilingue qui peut être à l'origine de la qualité jugée inacceptable des traductions.

Citons, en dernier lieu, la thèse de doctorat de Jääskeläinen, parue en 1999, dans laquelle l'auteur consacre une partie à l'étude de l'utilisation de dictionnaires chez des sujets représentant différents niveaux d'expérience de la traduction. L'auteur insiste à juste titre sur le fait que l'utilisation raisonnée de dictionnaires est un signe de professionnalisme plutôt que d'ignorance ${ }^{2}$ et observe que (a) les différences interindividuelles concernant la préférence pour une source d'information sont particulièrement grandes chez le groupe des traducteurs professionnels; (b) seuls les traducteurs professionnels utilisent des dictionnaires spécialisés pour résoudre des problèmes terminologiques; (c) les traducteurs non professionnels («educated laymen») utilisent une gamme de sources d'information moins large que les traducteurs professionnels et les étudiants en traduction. En examinant une nouvelle fois la relation entre le type de dictionnaires utilisés et la qualité des traductions, l'auteur observe que (a) les sujets ayant rendu des traductions jugées de «bonne qualité » ou de "moyenne qualité» affichent le taux de consultation de sources d'information le plus élevé; (b) ceux du groupe «bonne qualité» utilisent avant tout des dictionnaires monolingues pour venir à bout d'un problème ( $f i r s t$ look-ups»), alors que c'est l'inverse pour le groupe «faible qualité ». À l'instar de Englund Dimitrova et Jonasson (à paraître), Jääskeläinen (1999) en conclut que la préférence pour un type de dictionnaire (monolingue $v s$ bilingue) peut jouer un rôle dans la réussite ou l'échec relatifs d'une tâche de traduction. L'auteur calcule également ce qu'elle appelle «depth of research» (profondeur de la recherche), à savoir le nombre de consultations auquel donne lieu la recherche d'un équivalent dans la langue cible. Nous pensons que le terme «depth of research» n'est pas très heureux. En effet, il laisse supposer que plus un sujet consulte de sources d'information pour résoudre un problème, plus il travaille à un niveau sémantique profond, alors qu'un chiffre élevé peut simplement être le résultat de plusieurs consultations infructueuses. Jääskeläinen (1999) observe que ce coefficient est plus élevé chez les traducteurs professionnels que chez les traducteurs non professionnels, et que le coefficient du groupe des étudiants débutants se situe plus ou moins au même niveau que celui des traducteurs professionnels.

Si ces études ont permis de mieux comprendre le rôle que joue la consultation de sources d'information en traduction, nous avons néanmoins pu identifier les problèmes méthodologiques suivants:

- Choix de populations étrangères aux préoccupations des traductologues (des étudiants en langues étrangères et non en traduction).

- Validité écologique limitée de par (a) le choix du texte, souvent peu représentatif du travail prototypique du traducteur, ou, à la lumière des verbalisations des sujets et de la qualité de leurs traductions écrites, trop difficile pour eux; (b) le lieu d'observation qui est souvent la place de travail du chercheur et non celui du sujet; (c) l'impossibilité pour les sujets d'avoir accès à leurs sources d'information habituelles; (d) la création d'un cadre d'observation envahissant du fait du recours à la réflexion parlée d'une part, et à l'enregistrement du sujet sur vidéo d'autre part. 
- Description insuffisante de la démarche méthodologique (caractéristiques des sujets, lieu et conditions d'observation, procédure standardisée, etc.) qui rend difficile la comparaison des données.

- Erreurs dans l'interprétation des données dues à l'attribution de liens de cause à effet souvent plausibles, mais inadmissibles dans le cadre d'études corrélationnelles. Il s'agit ici du lien entre la préférence pour un dictionnaire (monolingue $v s$ bilingue) et la qualité de la traduction.

Afin de mieux comprendre certains mécanismes qui se sont dégagés des études décrites ci-dessus et d'y apporter les modifications méthodologiques jugées nécessaires, le présent travail avait trois objectifs :

- La création d'un cadre d'observation naturel permettant aux sujets (étudiants en traduction et traducteurs professionnels) de travailler à leur poste de travail habituel et avec les sources d'information qu'ils ont coutume d'utiliser;

- L'analyse du lien entre l'expérience de la traduction, la préférence pour un certain type de source d'information (en particulier, dictionnaires monolingues $v s$ dictionnaires bilingues) et la qualité de la traduction;

- L'analyse de l'utilisation de sources d'information non imprimées (dictionnaires électroniques, fonctions dans le traitement de texte, référence à des experts humains).

Nous sommes conscient des limites de notre étude, en particulier du nombre restreint de sujets participant à notre étude. En plus, la manière dont les sujets font appel à des sources d'information varie sûrement en fonction du degré de familiarité avec le genre de texte à traduire. Si nos résultats ne peuvent faire l'objet d'une généralisation, il sera néanmoins intéressant de les confronter aux analyses faites jusqu'ici dans ce domaine.

\section{Méthode}

\subsection{Sujets}

Nous avons demandé à trois étudiants de l'École de traduction et d'interprétation (ETI) de l'Université de Genève de traduire un texte de l'anglais vers l'allemand. Il s'agissait d'étudiants en première année de formation. Afin d'éviter une trop grande hétérogénéité au sein de chaque groupe en ce qui concerne les connaissances de l'anglais et l'expérience de la traduction, nous avons demandé à l'enseignante du cours de traduction anglais-allemand de nous indiquer trois étudiants ayant plus ou moins le même niveau. De plus, tous les étudiants devant passer un test d'admission avant d'entrer dans cette École, nous nous attendions à ce qu'il n'y ait pas trop de variabilité au départ.

Le deuxième groupe était composé de trois traducteurs professionnels qui ont tous suivi leur formation à l'ETI. Ils avaient entre 4 et 8 ans d'expérience professionnelle, et la traduction représentait leur principale source de revenu au moment où nous avons fait les observations. Voici le tableau illustrant les caractéristiques de base des sujets (tous les noms sont fictifs): 
TABLEAU 1

Caractéristiques de la population

\begin{tabular}{|l|l|l|l|l|l|l|}
\hline Nom & Nicolas & Olivier & Petra & Lara & Laurent & Sonja \\
\hline Statut & $\begin{array}{l}\text { Étudiant } \\
1^{\text {re }} \text { année }\end{array}$ & $\begin{array}{l}\text { Étudiant } \\
1^{\text {re }} \text { année }\end{array}$ & $\begin{array}{l}\text { Étudiante } \\
1^{\text {re }} \text { année }\end{array}$ & Traductrice & Traducteur & Traductrice \\
\hline Groupe d'âge & $20-30$ & $20-30$ & -20 & $31-40$ & $20-30$ & $31-40$ \\
\hline $\begin{array}{l}\text { Combinaison } \\
\text { linguistique }\end{array}$ & $\begin{array}{l}\text { Allemand } \\
\text { Anglais } \\
\text { Français }\end{array}$ & $\begin{array}{l}\text { Allemand } \\
\text { Français } \\
\text { Anglais }\end{array}$ & $\begin{array}{l}\text { Allemand } \\
\text { Anglais } \\
\text { Français }\end{array}$ & $\begin{array}{l}\text { Allemand } \\
\text { Français } \\
\text { Anglais } \\
\text { Espagnol }\end{array}$ & $\begin{array}{l}\text { Allemand } \\
\text { Français } \\
\text { Anglais } \\
\text { Italien }\end{array}$ & $\begin{array}{l}\text { Allemand } \\
\text { Français } \\
\text { Anglais }\end{array}$ \\
\hline Spécialisation & - & - & - & $\begin{array}{l}\text { Droit, } \\
\text { économie, } \\
\text { industrie }\end{array}$ & $\begin{array}{l}\text { Banque, } \\
\text { finance, } \\
\text { droit }\end{array}$ & $\begin{array}{l}\text { Droits } \\
\text { d'auteur, } \\
\text { journalisme }\end{array}$ \\
\hline
\end{tabular}

NOTE

Tous les sujets sont de langue maternelle allemande et travaillent à partir des langues mentionnées vers leur langue maternelle.

\subsection{Matériau et mandat de traduction}

Nous reproduisons en annexe le texte choisi pour cette étude. Il a été choisi d'un commun accord entre les membres $\mathrm{du}$ " Process team ${ }^{3}$ » qui regroupe plusieurs chercheurs actifs dans le domaine de l'analyse des processus de traduction. Les caractéristiques du texte sont les suivantes:

- Longueur: relativement court (169 mots);

- Source: magazine vendu dans la rue par des sans-abri, en Grande-Bretagne;

- Sujet: description du concept du magazine;

- Genre de texte et fonction: informatif, fonction référentielle (Nord 1997);

- Difficultés: relatives au vocabulaire et aux conventions de texte;

- Mandat: nous avons dit aux sujets que leur traduction était nécessaire au lancement d'une initiative similaire en Suisse alémanique. Le client suisse allemand supposé devait pouvoir se faire une idée des expériences de ce genre déjà faites à l'étranger.

\subsection{Lieu et conditions d'observation}

Notre objectif étant d'observer la façon dont les étudiants en traduction et les traducteurs professionnels utilisent les sources d'information, nous avons conduit les observations à leur place de travail habituelle. Ainsi, tous les sujets avaient accès aux sources d'information dont ils se servent d'habitude. Nous avons également mis à leur disposition un petit corpus de textes parallèles, c'est-à-dire des textes originaux rédigés en allemand et couvrant un sujet similaire (journal vendu par des chômeurs dans les rues de Zurich).

\subsection{Procédure}

Nous avons suivi une procédure standardisée afin d'éviter que des variables confondantes ne viennent brouiller les différences réelles entre les sujets. Tous les sujets ont d'abord reçu une feuille contenant des informations sur les objectifs généraux de l'expérience. Puis nous avons organisé une brève séance de préparation pour nous 
assurer qu'ils avaient bien compris la consigne. C'est seulement ensuite qu'ils ont reçu le texte à traduire et le mandat. Pendant que les sujets traduisaient, nous avons noté nos observations sur la façon dont ils s'y prenaient dans un carnet de bord. Nous avons également noté toute consultation de sources d'information. Quand ils ont terminé la traduction, nous avons arrêté l'enregistrement, sauvegardé la traduction sur disquette et leur avons demandé de remplir un questionnaire afin de récolter des informations concernant leur formation, leur expérience de la traduction, leurs impressions concernant la situation de réflexion parlée, et l'usage qu'ils font habituellement des sources d'information dans leur travail. Après avoir récolté toutes ces informations par écrit, nous avons réalisé une interview dans laquelle nous avons posé des questions plus spécifiques sur le genre de textes et le mandat que les étudiants reçoivent dans les cours de traduction ou, dans le cas des traducteurs professionnels, qu'ils recevaient à l'époque. Nous avons terminé la séance en leur donnant un compte rendu de l'expérience et de son suivi. Tous les sujets ont reçu la somme de CHF 20 - pour leur participation.

\section{Résultats}

Nous avons eu l'occasion de discuter des aspects qualitatifs de l'utilisation de sources d'information dans le cadre d'un autre travail (Künzli, 2000). C'est pourquoi nous allons nous concentrer ici sur une analyse quantitative des données recueillies par l'observation participante (carnet de bord).

Voici, en premier lieu, un résumé des répartitions en fonction de la source d'information consultée et du nombre total de consultations pour chaque sujet.

TABLEAU 2

Nombre de consultations (en nombres absolus)

\begin{tabular}{|l|c|c|c|c|c|c|c|c|c|c|}
\hline SUJETS & MOGLS & MOGLC & MO SLC & BIG & BIS & ENC & TP & GRA & JOU & SOMME \\
\hline Nicolas (É) & 11 & - & - & 9 & - & - & - & 1 & - & 21 \\
\hline Olivier (É) & 5 & 2 & - & 8 & - & - & - & - & - & 15 \\
\hline Petra (É) & 1 & - & - & 14 & - & - & - & - & - & 15 \\
\hline Lara (T) & 2 & 1 & - & 2 & 3 & - & 3 & 1 & 1 & 13 \\
\hline Laurent (T) & 2 & 3 & 1 & 9 & 2 & - & 1 & - & - & 18 \\
\hline Sonja (T) & - & - & - & 9 & - & 2 & 1 & 2 & 1 & 15 \\
\hline
\end{tabular}

NOTE

Liste des abréviations:

É = étudiant en première année

$\mathrm{T}=$ traducteur professionnel

MOGLS : dictionnaire monolingue général de langue source MOGLC: dictionnaire monolingue général de langue cible

MOSLC: dictionnaire monolingue spécialisé de langue cible
BIG : dictionnaire bilingue général BIS : dictionnaire bilingue spécialisé ENC: encyclopédie TP : texte parallèle GRA : grammaire JOU : journal

Nous proposons maintenant une synthèse des mêmes résultats, regroupés cette foisci en fonction des trois principales catégories de sources utilisées. 
TABLEAU 3

Nombre de consultations selon les trois principales catégories de sources (en nombres absolus et en pourcentages)

\begin{tabular}{|c|c|c|c|c|c|c|c|}
\hline \multirow[t]{2}{*}{ Sujets } & \multicolumn{2}{|c|}{$\begin{array}{c}\text { Dictionnaires } \\
\text { bilingues }\end{array}$} & \multicolumn{2}{|c|}{$\begin{array}{l}\text { Dictionnaires } \\
\text { monolingues }\end{array}$} & \multicolumn{2}{|c|}{ Autres } & \multirow[t]{2}{*}{ Somme } \\
\hline & $\mathrm{N}$ & $\%$ & $\mathrm{~N}$ & $\%$ & $\mathrm{~N}$ & $\%$ & \\
\hline Nicolas (É) & 8 & 38 & 12 & 57 & 1 & 5 & 21 \\
\hline Olivier (É) & 8 & 53 & 7 & 47 & - & - & 15 \\
\hline Petra (É) & 14 & 93 & 1 & 7 & - & - & 15 \\
\hline Lara $(\mathrm{T})$ & 5 & 38 & 3 & 24 & 5 & 38 & 13 \\
\hline Laurent $(\mathrm{T})$ & 11 & 61 & 6 & 33 & 1 & 6 & 18 \\
\hline Sonja $(\mathrm{T})$ & 9 & 60 & - & - & 6 & 40 & 15 \\
\hline
\end{tabular}

Le tableau suivant donne le nombre de sources consultées par chacun des sujets.

TABLEAU 4

Nombre de sources consultées (en nombres absolus)

\begin{tabular}{|c|c|}
\hline Sujets & Nombre de sources consultées \\
\hline Nicolas (É) & 3 \\
\hline Olivier $(\hat{\mathrm{E}})$ & 3 \\
\hline Petra (É) & 2 \\
\hline Lara $(\mathrm{T})$ & 7 \\
\hline Laurent $(\mathrm{T})$ & 6 \\
\hline Sonja $(\mathrm{T})$ & 5 \\
\hline
\end{tabular}

En nous référant aux tableaux 2, 3 et 4, nous notons une différence réelle entre les deux groupes par rapport au type et au nombre de sources consultées. En effet, les étudiants consultent en moyenne 2,7 sources différentes, contre 6,3 pour les traducteurs professionnels. Notons en particulier que c'est l'utilisation de sources autres que les dictionnaires monolingues et bilingues qui distingue les deux groupes. En effet, chez 2 traducteurs sur 3, l'utilisation d'encyclopédies, de textes parallèles, de grammaires et de journaux représente plus d'un tiers de l'ensemble des consultations, alors que ces types de sources n'intéressent qu'un seul sujet du groupe des étudiants. Ajoutons également que la seule catégorie représentée chez tous les traducteurs, et absente chez tous les étudiants, est celle des textes parallèles.

Nous notons, d'autre part, que le dictionnaire bilingue constitue, somme toute, l'outil le plus utilisé par les sujets. Ainsi, le taux de consultation du dictionnaire bilingue est de $38 \%$ au minimum, cette proportion atteignant $93 \%$ pour une étudiante. En moyenne, le dictionnaire bilingue représente $57 \%$ de toutes les consultations de sources d'information. Le dictionnaire monolingue arrive en deuxième position avec $28 \%$, alors que le taux des autres sources utilisées ne dépasse pas 15\%. Précisons cependant que les fluctuations entre les sujets sont assez importantes. Ainsi, pour le dictionnaire monolingue, la variation du taux entre les six sujets est de $57 \%$, de $55 \%$ pour le dictionnaire bilingue et de $40 \%$ pour les autres sources utilisées. Il est cependant intéressant de noter que dans notre travail, les étudiants utilisent plus les dictionnaires 
monolingues que les traducteurs professionnels, contrairement à ce qu'ont mis en évidence d'autres travaux cités. En effet, la proportion de consultations de dictionnaires monolingues atteint une moyenne de 37\% chez le groupe des étudiants, alors qu'elle n'est que de $19 \%$ chez les traducteurs professionnels qui semblent compenser ce chiffre bas par l'utilisation d'autres sources d'information monolingues, comme des textes parallèles ou des grammaires. En ce qui concerne le nombre total de consultations, nous notons peu de différences entre les deux groupes: la moyenne s'élève à 15,3 pour le groupe des traducteurs et à 17 pour celui des étudiants.

Relevons encore un cas de figure surprenant, à savoir l'absence totale d'utilisation de dictionnaires monolingues chez Sonja, traductrice professionnelle, dont la traduction est la meilleure avec celle de Laurent (cf. tableau 8). De même, nous observons une nette préférence pour l'utilisation de dictionnaires bilingues et par conséquent une absence presque totale de recours à des dictionnaires monolingues chez Petra, étudiante en première année, dont la traduction écrite est jugée aussi bonne que celle de Lara, traductrice professionnelle qui a recours au plus grand nombre de sources d'information. Un phénomène identique, mais inversé, s'observe chez Nicolas, étudiant en première année, qui préfère travailler avec des dictionnaires monolingues, et chez qui l'on observe le taux le plus élevé de consultations, mais dont la traduction est considérée comme la moins bonne.

Venons-en maintenant à l'étude de la source d'information vers laquelle les sujets se tournent en premier lieu pour résoudre un problème ( $f$ first look-ups»). L'étude comprend également le nombre total d'éléments du texte source ayant suscité une consultation.

TABLEAU 5

Première source d'information consultée ( first look-ups»)

\begin{tabular}{|c|c|c|c|c|c|c|c|}
\hline Sujets & Éléments & Première s & urce d'inf & mation co & sultée & & \\
\hline Nicolas (É) & 12 & $\begin{array}{l}\text { MOGLS } \\
9(75 \%)\end{array}$ & $\begin{array}{c}\text { BIG } \\
3(25 \%)\end{array}$ & & & & \\
\hline Olivier (É) & 11 & $\begin{array}{c}\text { BIG } \\
7(64 \%)\end{array}$ & $\begin{array}{l}\text { MOGLS } \\
3(27 \%)\end{array}$ & $\begin{array}{c}\text { MOGLC } \\
1(9 \%)\end{array}$ & & & \\
\hline Petra (É) & 14 & $\begin{array}{c}\text { BIG } \\
14(100 \%)\end{array}$ & & & & & \\
\hline Lara $(\mathrm{T})$ & 7 & $\begin{array}{c}\mathrm{TP} \\
2(30 \%)\end{array}$ & $\begin{array}{l}\text { MOGLS } \\
1(14 \%)\end{array}$ & $\begin{array}{c}\text { MOGLC } \\
1(14 \%)\end{array}$ & $\begin{array}{c}\text { BIG } \\
1(14 \%)\end{array}$ & $\begin{array}{c}\text { JOU } \\
1(14 \%)\end{array}$ & $\begin{array}{c}\text { GRA } \\
1(14 \%)\end{array}$ \\
\hline Laurent $(\mathrm{T})$ & 9 & $\begin{array}{c}\text { BIG } \\
8(89 \%)\end{array}$ & $\begin{array}{c}\text { BIS } \\
1(11 \%)\end{array}$ & & & & \\
\hline Sonja $(\mathrm{T})$ & 11 & $\begin{array}{c}\text { BIG } \\
8(73 \%)\end{array}$ & $\begin{array}{c}\text { GRA } \\
1(9 \%)\end{array}$ & $\begin{array}{c}\text { JOU } \\
1(9 \%)\end{array}$ & $\begin{array}{c}\text { TP } \\
1(9 \%)\end{array}$ & & \\
\hline
\end{tabular}

NOTE

Liste des abréviations: voir note en bas du tableau 2 .

En nous référant au tableau 5 , nous pouvons constater que dans la plupart des cas, 4 sujets sur 6 consultent avant tout un dictionnaire bilingue pour venir à bout d'un problème. Chez les deux sujets restants, le dictionnaire bilingue arrive en 
deuxième position. Somme toute, les sujets se tournent d'abord vers les dictionnaires bilingues dans $66 \%$ des recherches effectuées. La catégorie des dictionnaires monolingues arrive en deuxième position avec $23 \%$, suivie par la catégorie «autres sources » qui représente $11 \%$ des comportements observés. Relevons par ailleurs un fait surprenant: pour les étudiants, les dictionnaires monolingues représentent plus souvent la première source consultée que pour les traducteurs professionnels. Par conséquent, si le dictionnaire bilingue est prédominant pour ce critère, il ne nous est pourtant pas possible d'affirmer, comme ce qui semble ressortir d'autres recherches, que les sources autres que les dictionnaires bilingues sont utilisées uniquement dans les cas où l'utilisation d'un dictionnaire bilingue s'est avérée improductive.

Par ailleurs, force est de constater l'importante hétérogénéité à l'intérieur de chacun des groupes. En effet, alors que Petra, étudiante en première année, commence chaque recherche par le recours à un dictionnaire bilingue, Nicolas, son condisciple, affiche une nette préférence pour le dictionnaire monolingue qui représente $75 \%$ des premières consultations. Les résultats varient également à l'intérieur du groupe des traducteurs. Ainsi, Laurent commence toutes ses recherches par la consultation d'un dictionnaire bilingue, alors que le même chiffre ne dépasse pas les 14\% chez Lara qui, elle, ne présente pas vraiment de préférence pour un certain type de source. D’autre part, nous pouvons relever que les étudiants font d'abord appel à 2 types d'outils dictionnaires monolingues de langue source et de langue cible d'un côté, et dictionnaires bilingues de l'autre - , contre 5 types d'outils pour les traducteurs professionnels. Il est également intéressant de signaler que les traductions effectuées par Petra et Lara, qui affichent des comportements radicalement opposés pour le critère de la première source consultée, sont jugées qualitativement équivalentes.

Nous proposons maintenant une synthèse des résultats obtenus pour chacun des sujets concernant l'utilisation de sources d'information non imprimées.

TABLEAU 6

Utilisation de sources d'information non imprimées

\begin{tabular}{|l|c|c|c|}
\hline Sujets & $\begin{array}{c}\text { Dictionnaires } \\
\text { électroniques }\end{array}$ & $\begin{array}{c}\text { Vérificateur d'orthographe/ } \\
\text { autres fonctions dans WORD }\end{array}$ & Experts humains \\
\hline Nicolas (É) & Non & Oui & Non \\
\hline Olivier (É) & Non & Non & Non \\
\hline Petra (É) & Non & Non & Oui \\
\hline Lara (T) & Oui & Oui & Non \\
\hline Laurent (T) & Non & Oui & Non \\
\hline Sonja (T) & Non & Oui & \\
\hline
\end{tabular}

NOTE

Nous avons renoncé à effectuer des calculs en nombres absolus étant donné que la consultation de sources d'information non imprimées ne relève pas toujours d'un comportement matériellement observable.

À la lecture du tableau 6, nous pouvons observer une certaine différence entre les deux groupes. Cette différence est encore plus nette si nous tenons compte de toutes les données recueillies (données obtenues par le biais de l'observation participante, des interviews et des questionnaires). Ainsi, nous sommes en droit d'affirmer que seuls les traducteurs professionnels font mention de dictionnaires électroniques 
comme sources d'information possibles, qu'ils sont également les seuls à exploiter de manière systématique les outils d'aide contenus dans les traitements de texte (vérificateur d'orthographe, synonymie, etc.), et à souligner l'importance d'un réseau d'experts humains à qui faire appel si la consultation des sources d'information imprimées s'avère improductive. Si la différence de comportement entre les étudiants en traduction et les traducteurs professionnels va presque de soi pour le critère des experts humains - les étudiants ne sont pas encore habitués à se voir comme un maillon de la chaîne qui va de l'intermédiaire chargé de faire traduire le texte au destinataire final de la traduction - les résultats obtenus pour les deux autres critères, à savoir l'utilisation de dictionnaires électroniques sont plus difficiles à comprendre. Nous y reviendrons dans la discussion.

Nous proposons maintenant, pour chaque sujet, une synthèse de la proportion entre le nombre d'éléments recherchés et le nombre de consultations, afin de calculer ce que nous appellerons le «coefficient de consultation» (cf. aussi Jääskeläinen, 1999).

TABLEAU 7

Coefficient de consultation

\begin{tabular}{|c|c|c|c|}
\hline Sujets & Éléments recherchés & Consultations & Proportion \\
\hline Nicolas (É) & 12 & 21 & 1,8 \\
\hline Olivier (É) & 11 & 15 & 1,4 \\
\hline Petra (É) & 14 & 15 & 1,1 \\
\hline Lara $(\mathrm{T})$ & 7 & 13 & 1,9 \\
\hline Laurent $(\mathrm{T})$ & 9 & 18 & 2,0 \\
\hline Sonja $(\mathrm{T})$ & 11 & 15 & 1,4 \\
\hline
\end{tabular}

Pour ce critère, nous observons une nouvelle fois que les fluctuations entre les sujets prennent des dimensions assez importantes. Si nous nous référons d'abord au résultat de Petra, étudiante en première année, nous constatons qu'elle utilise deux dictionnaires pour résoudre un problème seulement 1 fois sur 14, alors que Laurent, traducteur professionnel, manifeste une claire tendance à vérifier le résultat d'une consultation d'un dictionnaire dans d'autres sources, les autres sujets se situant entre ces deux extrêmes. Si un coefficient de consultation élevé peut effectivement être l'indicateur d'une certaine méfiance à l'égard de la fiabilité d'une source d'information, et donc de la volonté de trouver confirmation d'un résultat de recherche dans au moins une deuxième source, elle peut tout aussi bien être le résultat d'une tentative de recherche infructueuse, entraînant la consultation d'autres sources d'information dans le but de trouver l'information recherchée. À ce titre, les comportements respectifs de Laurent et de Lara sont significatifs. Tous deux se voient obligés de consulter un dictionnaire pour rechercher la signification de l'abréviation anglaise «SAE» («Stamped Addressed envelope»). Laurent infère la signification correcte à partir du contexte avant de consulter une source d'information, trouve confirmation dans le premier dictionnaire consulté, mais décide néanmoins d'avoir recours à un deuxième dictionnaire pour trouver une nouvelle confirmation. Soulignons également que cette tendance à chercher confirmation est un signe caractéristique du travail de Laurent qui explique en partie son coefficient de consultation élevé. Lara, quant à elle, ne 
connait pas non plus la signification de «SAE» et se trouve donc obligée de consulter non moins de 7 dictionnaires pour trouver la solution à ce problème de traduction, ce qui fait grimper son coefficient de consultation.

Tout en étant conscient que des comportements extrêmes d'un côté ou de l'autre de l'échelle se répercutent massivement sur la moyenne d'un petit échantillon, nous nous permettons de relever qu'en moyenne, le coefficient de consultation est plus élevé dans le groupe des traducteurs professionnels $(1,8)$ que dans celui des étudiants $(1,4)$. Pourtant, chez les étudiants, la moyenne du nombre d'éléments posant problème et suscitant une consultation de dictionnaires et la moyenne du nombre total de consultations sont plus élevées.

De plus, force est de constater l'absence de corrélation entre le coefficient de consultation et la qualité de la traduction. La bonne performance de Petra, qui présente par ailleurs le coefficient de consultation le plus bas, et la qualité médiocre de la traduction de Nicolas, qui affiche le coefficient de consultation le plus élevé des étudiants, interdisent toute interprétation des données fondée uniquement sur la notion de «depth of research» (Jääskeläinen 1999).

Voici, en dernier lieu, le résultat de l'évaluation de la qualité des traductions, effectuée par deux correcteurs indépendants, qui, lors de la remise de leur classement, n'étaient au courant ni de l'appartenance des sujets à un groupe ou l'autre, ni du jugement de l'autre correcteur. Les deux correcteurs sont des enseignants en traduction et ont effectué leur évaluation sur la base des informations contenues dans le mandat de traduction fourni aux sujets.

TABLEAU 8

Évaluation de la qualité des traductions

\begin{tabular}{|c|c|c|c|}
\hline Sujets & Correcteur A & Correcteur B & Moyenne \\
\hline Nicolas (É) & 6 & 6 & 6 \\
\hline Olivier (É) & 5 & 5 & 5 \\
\hline Petra (É) & 3 & 4 & 3.5 \\
\hline Lara $(\mathrm{T})$ & 4 & 3 & 3.5 \\
\hline Laurent $(\mathrm{T})$ & 2 & 1 & 1.5 \\
\hline Sonja $(\mathrm{T})$ & 1 & 2 & 1.5 \\
\hline
\end{tabular}

NOTE

$1=$ meilleure traduction $/ 6=$ moins bonne traduction.

Les évaluations des traductions faites par les deux correcteurs se rejoignent. Elles vont toutes les deux dans le sens d'une forte corrélation positive entre le degré d'expérience de la traduction et la qualité de la traduction. Le groupe des traducteurs arrive donc en tête du classement, alors que les traductions faites par les étudiants sont jugées moins bonnes, exception faite de celle rendue par Petra. En effet, cette étudiante est la seule à rendre une traduction jugée acceptable et aussi bonne que celle de Lara, traductrice professionnelle.

Insistons également sur certains liens intéressants entre la qualité de la traduction et les résultats obtenus pour d'autres critères. Ainsi, pour la qualité de la traduction, les deux sujets qui arrivent en tête du classement — Sonja et Laurent — font largement appel à des dictionnaires bilingues au détriment d'autres sources d'information comme les dictionnaires monolingues, tout en ayant recours, à la différence des 
étudiants, à un nombre élevé d'autres sources d'information pour résoudre des problèmes plus spécifiques (par exemple, des grammaires pour des problèmes de déclinaisons ou des journaux pour des calculs de taux de change entre le franc suisse et la livre sterling, etc.). Par ailleurs, Sonja et Laurent commencent leurs recherches par la consultation de dictionnaires bilingues, et utilisent les vérificateurs d'orthographe, de grammaire et de synonymie contenus dans leur traitement de texte. C'est Nicolas, étudiant en traduction, qui arrive en fin de classement. Cet étudiant en traduction est le sujet qui fait le plus usage de dictionnaires monolingues. 3 fois sur 4 , il commence ses consultations de dictionnaires par le recours à un dictionnaire monolingue. C'est aussi le seul étudiant à utiliser un vérificateur d'orthographe, et il affiche un coefficient de consultation plus élevé que ceux de ses deux condisciples. Olivier arrive en avant-dernière position. Après Nicolas, c'est le sujet qui a le plus souvent recours aux dictionnaires monolingues. Dans plus d'un tiers des cas, il commence ses recherches d'un équivalent en langue cible par le recours à un dictionnaire monolingue. Enfin, Petra et Lara sont classées ex-æquo au troisième rang et se distinguent beaucoup en ce qui concerne l'utilisation des sources d'information : Petra, étudiante en traduction, préfère nettement les dictionnaires bilingues, alors que Lara affiche la répartition la plus équilibrée entre les différentes sources d'information; Petra a recours au nombre de sources le plus bas de tous les sujets, alors que Lara l'emporte pour ce même critère; Petra commence toutes ses recherches par le recours à un dictionnaire bilingue, alors que ce taux n'est que de 14\% chez Lara; Petra n'utilise aucune source d'information non imprimée, alors que toutes les catégories de sources recensées sont représentées chez Lara; enfin, Petra présente le coefficient de consultation le moins élevé, alors que Lara arrive en deuxième position pour ce critère. Ce cas de figure tout à fait intéressant, qui est lié à deux comportements radicalement opposés, montre clairement qu'en l'état actuel de nos connaissances des mécanismes intervenant dans le processus de traduction, il faut se garder de conclure trop vite à des constantes dans le comportement traduisant. En effet, notre compréhension de l'interaction entre les différentes variables influant sur le processus de traduction, ou étant liée à la qualité de la traduction, est encore limitée.

\section{Discussion}

Nous allons maintenant confronter nos résultats aux autres études concernant l'utilisation des sources d'information en traduction, discuter des implications de notre étude et terminer par un survol des pistes qui pourraient donner lieu à des recherches ultérieures.

Nous avons vu que les sujets de Krings (1986), qui apprenaient le français comme langue étrangère, se caractérisent par l'utilisation quasi exclusive de dictionnaires bilingues et semblent peu se soucier de la qualité de la traduction rendue. Cela n'est pas le cas de nos sujets. En effet, le comportement de nos étudiants en traduction donne à penser qu'ils comprennent vite que traduire, c'est produire un texte cible acceptable et compréhensible, et que cela ne relève pas seulement d'un test d'aptitude en L2, même si les résultats obtenus par deux de nos sujets, Nicolas et Olivier, montrent que la compréhension de la fonction de la traduction ne se traduit pas forcément par un succès matériel, à savoir une traduction de qualité. Toutefois, ils se distinguent des sujets de Krings (1986) par leur recours à une gamme plus large 
de sources d'information pour résoudre les différents problèmes faisant obstacle à la production d'une traduction jugée acceptable. Le comportement de Petra, troisième membre de notre groupe d'étudiants, qui utilise un dictionnaire bilingue pour résoudre un problème dans $93 \%$ des cas, ne remet pas forcément en cause cette différence fondamentale entre étudiants en L2 et étudiants en traduction, car contrairement aux sujets de Krings (1986) qui, à la lumière de leurs problèmes de compréhension, auraient pu profiter d'une utilisation de dictionnaires monolingues, Petra n'en ressent peut-être pas le besoin, comme le montre la qualité de sa traduction.

Jääskeläinen (1989) constate que les étudiants débutants en traduction affichent une préférence pour le travail avec des dictionnaires bilingues, alors que dans notre groupe d'étudiants, Nicolas préfère travailler avec le dictionnaire monolingue, outil qui représente même $47 \%$ des consultations chez Olivier. Nous constatons la même différence pour le critère de la première source consultée ( «first look-ups»). En effet, Jääskeläinen (1999) observe que les étudiants débutants se tournent en premier lieu vers des dictionnaires bilingues, alors que dans notre groupe, Nicolas utilise un dictionnaire monolingue avant toute autre source dans 3 cas sur 4 . Finalement, nous n’avons pas pu mettre en évidence, contrairement à Jääskeläinen (1989), une corrélation positive entre la qualité de la traduction et la préférence du dictionnaire monolingue. Au contraire: nous sommes proches d'une corrélation négative, étant donné qu'arrivent en tête des jugements de la qualité des traductions deux sujets qui privilégient le travail avec des dictionnaires bilingues, dont un — Sonja — n'a même jamais recours à un dictionnaire monolingue, alors que Nicolas et Olivier ferment la marche et ont le plus souvent recours à des dictionnaires monolingues! Cette variabilité des résultats d'une étude à l'autre souligne une nouvelle fois la complexité de l'acte traduisant dont l'analyse des différents facteurs prendra encore beaucoup de temps. En effet, comment expliquer que Nicolas et Olivier aient si souvent recours à des dictionnaires monolingues, contrairement à Petra et aux étudiants de Jääskeläinen (1989) ? Nous avons vu qu'il existe de la part de beaucoup d'enseignants en traduction une tendance à déconseiller l'usage de dictionnaires bilingues. Peut-être ces deux étudiants cherchaient-ils à mettre en pratique cette recommandation lors de leur participation à notre étude, sans toutefois pouvoir exploiter les informations contenues dans les dictionnaires monolingues de façon optimale (en raison de leurs connaissances insuffisantes en anglais?). Le fait que Petra ait grandi à l'étranger et fréquenté des écoles dont la langue d'enseignement était l'anglais expliquerait dès lors qu'elle n'ait pas besoin de consulter des dictionnaires monolingues en langue source pour résoudre des problèmes de compréhension et qu'elle choisisse en premier lieu des dictionnaires bilingues pour chercher des équivalents en langue cible.

Englund Dimitrova \& Jonasson (à paraître) constatent que les traducteurs professionnels privilégient le travail avec des dictionnaires monolingues, résultat que notre recherche ne confirme pas. Une nouvelle fois, nos résultats se distinguent également quant à la corrélation éventuelle entre la préférence d'une certaine source d'information et la qualité de la traduction. Les auteurs observent une corrélation positive entre l'usage de dictionnaires monolingues et la qualité de la traduction, alors qu'arrivent en tête de notre jugement de la qualité deux sujets préférant travailler avec le dictionnaire bilingue. Rappelons toutefois que dans l'étude de Englund Dimitrova \& Jonasson (à paraître), les deux sujets ayant rendu les meilleures traductions sont non seulement ceux qui préfèrent travailler avec des dictionnaires monolingues, mais 
sont également des traducteurs professionnels. Il est donc possible que l'expérience de la traduction contribue à la préférence affichée pour un certain type de source d'information et explique la meilleure qualité de la traduction. Nous nous demandons d'ailleurs si la préférence que les traducteurs professionnels manifestent dans les études citées pour le dictionnaire monolingue ne relève pas d'un artefact de la situation d'observation. En effet, contrairement à notre étude et à celle de Nord (1999), où les traducteurs professionnels ont eu l'occasion de travailler à leur place de travail habituelle et avec les sources d'information qu'ils ont coutume d'utiliser, Englund Dimitrova \& Jonasson (à paraître) et Jääskeläinen (1999) ont conduit les observations à la place de travail du chercheur où les traducteurs professionnels n'ont pas accès à toutes leurs sources d'information habituelles. Cet état de fait ne peut que renforcer le sentiment qu'ont les sujets d'être observés et, partant, la tendance à vouloir deviner les attentes du chercheur en faisant preuve d'un comportement traduisant plus sophistiqué que d'habitude, c'est-à-dire en évitant d'avoir trop recours à des dictionnaires bilingues souvent jugés peu fiables par les enseignants en traduction et par les traductologues. Cette crainte des sujets d'être considérés comme peu consciencieux par le chercheur est sûrement plus présente chez les traducteurs professionnels que chez les étudiants en traduction, les premiers étant soucieux de ne pas «perdre la face».

En nous référant plus en détail au travail de Jääskeläinen paru en 1999, nous trouvons confirmation de certains de nos résultats. Ainsi, nous avons également pu constater que les traducteurs professionnels utilisent une gamme de sources d'information plus large, et qu'ils sont les seuls à utiliser des dictionnaires spécialisés. En revanche, nous ne pouvons toujours pas être d'accord avec les observations concernant la relation entre le type de source d'information utilisée et la qualité de la traduction. Contrairement à Jääskeläinen (1999), nous n'avons trouvé de corrélation positive ni entre le nombre de consultations et la qualité de la traduction d'une part, ni entre la préférence pour le travail avec des dictionnaires monolingues comme première source consultée et la qualité de la traduction d'autre part. Selon l'auteur, la corrélation positive entre le taux de consultation et la qualité de la traduction montrerait que pour un traducteur, le fait de savoir repérer les problèmes potentiels d'un texte lui permettrait d'assurer une certaine qualité de la traduction. Cette explication nous paraît tout à fait raisonnable. Or, dans notre étude, nous pourrions également expliquer le fait que les traducteurs professionnels affichent le nombre le moins élevé de consultations par leur souci de faire une utilisation optimale du temps qui leur est imparti. En Suisse, le traducteur étant payé dans la plupart des cas au nombre de mots traduits, chaque minute et chaque consultation qui exige du temps comptent. Il est dès lors impératif pour le traducteur d'éviter toute consultation qui ne soit pas jugée utile et potentiellement productive.

Nos résultats ont différentes implications. Sur le plan méthodologique, il nous paraît important de souligner le danger potentiel que représente l'établissement de liens de cause à effet dans des études corrélationnelles. En effet, au vu des résultats des études citées, on pourrait être tenté de conclure que la préférence pour le dictionnaire bilingue est à l'origine de la qualité médiocre de la traduction. Si cette conclusion est probable, elle ne saurait être tirée d'une étude purement corrélationnelle, où il convient de tenir compte du fait que deux variables - en l'occurrence, l'utilisation d'une source d'information et la qualité d'une traduction — peuvent être liées à leur 
tour à un troisième facteur, comme l'expérience de la traduction. Plutôt que de faire de la variable A (source d'information) la cause de la variable B (qualité de la traduction), et à la lumière des résultats obtenus dans les travaux cités et dans les nôtres, nous tendrions à affirmer que les variables $\mathrm{A}$ et $\mathrm{B}$ sont liées à un autre facteur, en l'occurrence, l'expérience de la traduction. Dans le cadre d'une étude expérimentale, il serait toutefois possible de vérifier l'hypothèse selon laquelle le type de source utilisée influence la qualité de la traduction. On pourrait ainsi manipuler la variable «accès aux sources d'information» et faire traduire un texte A à des sujets en leur permettant l'accès à un certain type de source, puis un texte B en leur refusant l'accès à cette même source.

Deuxièmement, nos résultats sont pertinents pour l'enseignement de la traduction. Ainsi, nous avons insisté à plusieurs reprises sur l'importance des différences entre les sujets pour ce qui est de l'utilisation des sources d'information. Nous avons vu en particulier qu'il est possible de produire une traduction acceptable en utilisant presque exclusivement des dictionnaires bilingues, source souvent considérée comme peu fiable dans les approches normatives ou pédagogiques de la traduction (par exemple Kussmaul, 1995; Snell-Hornby, 1995). Inversement, nous avons constaté que le recours à des dictionnaires monolingues ne garantit pas forcément la bonne qualité de la traduction. Dès lors, nous ne pouvons que donner raison à Nord (1999). Cette dernière désapprouve le fait qu'en traductologie, l'utilisation du dictionnaire monolingue soit souvent recommandée, tandis que celle du dictionnaire bilingue est critiquée. Au terme de son étude portant sur l'usage des sources d'information par les traducteurs professionnels, l'auteur arrive à la conclusion que les dictionnaires bilingues constituent un outil précieux et tout à fait valable pour chaque traducteur professionnel. Plutôt que de déconseiller l'usage d'un certain type d'outil, il importe, selon elle, d'offrir aux étudiants en traduction une introduction systématique à la lexicographie et à la terminologie pour qu'ils puissent faire le meilleur usage possible de toutes les sources d'information disponibles. Ajoutons à cet égard qu'il serait utile d'attirer l'attention des étudiants sur la nécessité de ne pas négliger les sources non imprimées, comme les dictionnaires électroniques, et toutes les fonctions offertes dans les traitements de texte. Rappelons également que dans notre étude, aucun étudiant n'a feuilleté les textes parallèles que nous avons mis à leur disposition et dont la consultation aurait permis de produire une traduction plus naturelle et plus idiomatique, alors que tous les traducteurs y ont eu recours. Dans la pratique, les textes parallèles comme source de documentation sont souvent plus précieux que n’importe quel dictionnaire, car ils offrent des équivalents en langue cible dépassant le niveau du mot et permettent la réalisation de traductions adaptées aux besoins du client. Afin de pouvoir simuler la réalité traduisante, il faudra cependant que les cours de traduction utilisent le plus souvent possible des textes que le traducteur professionnel rencontre effectivement. C'est rarement le cas, si l'on en juge par le recours massif qu'ont les départements de traduction à des articles de presse et de littérature dans les cours de traduction dite "générale». Le choix de textes peu représentatifs explique peut-être que les étudiants participant à des projets de recherche utilisent peu les dictionnaires électroniques, outils précieux pour la résolution de problèmes terminologiques. Rappelons enfin que l'on aurait tout intérêt à permettre l'usage de sources d'information lors des contrôles de connaissances, étant donné qu'elles constituent des instruments indispensables au travail du traducteur. 
Venons-en finalement aux pistes de recherche qui se dégagent pour l'avenir. Il est coutume d'insister à cet endroit sur la nécessité de transférer le plan d'observation à un autre groupe de sujets. En l'occurrence, nous sommes en présence d'une situation favorable. Nous avons déjà mentionné que le texte que nous avons donné à traduire aux sujets a été choisi d'un commun accord entre différents chercheurs actifs dans le domaine de l'analyse des processus de traduction. Si chaque chercheur a poursuivi ses propres objectifs en utilisant ce texte dans le cadre de ses projets de recherche, il est néanmoins possible de réexaminer tout le matériel recueilli à la lumière d'un seul critère, en l'occurrence l'usage que font les sujets des sources d'information. Le fait de pouvoir assurer la constance de la variable «texte source» et de suivre une procédure strictement standardisée permettra de réduire en partie le grand nombre de variables intervenant dans la traduction.

Mais la seule analyse des données issues du présent travail soulève un nombre important de questions qu'il serait sans doute intéressant d'étudier. Nous en citons une qui nous parait particulièrement pertinente. Dans notre analyse des protocoles de réflexion parlée, nous avons décelé des différences dans la façon dont les deux groupes - étudiants en traduction et traducteurs professionnels — utilisent le mandat de traduction: si les traducteurs professionnels s'y réfèrent à plusieurs reprises de manière explicite et s'en servent dans leurs processus de prise de décision, les deux étudiants dont les traductions étaient les moins bonnes semblent négliger le mandat pendant leur activité traduisante. De plus, en nous fondant sur l'analyse de la qualité des traductions, nous constatons la chose suivante: l'étudiant ayant rendu la traduction la moins bonne aurait pu éviter de nombreuses fautes s'il avait au préalable analysé les informations du mandat donné aux étudiants avant qu'ils ne commencent à traduire. Le mandat de traduction constituant en quelque sorte une source d'information de par les informations qu'il contient sur le destinataire potentiel, sur les attentes concernant le style ou sur le support sur lequel la traduction sera publiée, nous pensons qu'il serait particulièrement intéressant d'étudier le rôle qu'il joue dans les processus de traduction. Cela permettrait aussi d'apporter un complément empirique à cette notion particulièrement chère aux approches fonctionnalistes de la traduction.

\section{NOTES}

1. Cette étude a été financée par le Fonds commémoratif de la Banque centrale de Suède. Je tiens à remercier les membres de TRAPROS ( Translation Process Group in Sweden «) pour leur lecture critique d'une ancienne version de cet article. Je remercie également tous les sujets d'avoir participé à cette étude.

2. Notons cependant que dans beaucoup de départements de traduction, les étudiants n'ont pas droit aux dictionnaires lors de contrôles de connaissances.

3. Ce groupe a été fondé à l'initiative de Sonja Tirkkonen-Condit, Université de Savonlinna, Finlande.

\section{RÉFÉRENCES}

Englund Dimitrova, B. \& K. Jonasson (à paraître): "Translation Ability and Translatorial Competence: Expert and Novice Use of Dictionaries», in H. Kalverkämper \& B. Svane (Eds.), Translation and Interpreting. State and Perspectives. Proceedings from the HumboldtStockholm Symposium, Stockholm University, January 30-31, 1997.

Gile, D. (1995): Basic Concepts and Models for Interpreter and Translator Training, Amsterdam, John Benjamins. 
JäÄskeläinen, R. (1989) : «The Role of Reference Material in Professional vs. Non-Professional Translation: A Think-Aloud Protocol Study», in S. Tirkkonen-Condit \& S. Condit (Eds.), Empirical Studies in Translation and Linguistics, Joensuu, University of Joensuu, p. 175-200.

JäÄskeläInen, R. (1999): Tapping the Process: An Exploratory Study of the Cognitive and Affective Factors Involved in Translating, Joensuu, University of Joensuu publications in the Humanities, 22.

KRINGs, H. P. (1986): Was in den Köpfen von Übersetzern vorgeht, Tübingen, Narr.

Künzli, A. (2000): "The Role of Pragmatic Analysis in the Translation of Text-Type Conventions», in B. Englund Dimitrova (Ed.), Översättning och tolkning. Rapport från ASLA:s höstsymposium, Stockholm, 5-6 november 1998, Uppsala, ASLA, p. 207-218.

Kussmaul, P. (1995): Training the Translator, Amsterdam, John Benjamins.

Nord, B. (1999): 'Das zweisprachige Wörterbuch als Hilfsmittel bei der Übersetzung', In A. GiL, J. Haller, E. Steiner, \& H. Gerzymisch-Arbogast (Eds.), Modelle der Translation, Frankfurt a. M., Peter Lang, p. 375-390.

- (1997): Translating as a Purposeful Activity, Manchester, St. Jerome.

Schäffner, C. (1998) : «Parallel Texts in Translation», In L. Bowker, M. Cronin, D. Kenny, \& J. Pearson (Eds.), Unity in Diversity? Current Trends in Translation Studies, Manchester, St. Jerome, p. 83-90.

Snell-Hornby, M. (1995): Translation Studies. An Integrated Approach, Amsterdam, John Benjamins.

\footnotetext{
ANNEXE

Le texte source

"The Concept»

The Big Issue is sold by homeless, vulnerably housed and ex-homeless people who buy the magazine for $35 \mathrm{p}$ and sell it to the public for $80 \mathrm{p}$. All vendors receive training, sign a code of conduct and can be identified by Big Issue badges. The Big Issue is sold in London, Scotland, the North West, Bath, Brighton, Birmingham, Bristol, Ireland, Cardiff and the North East. The Big Issue also provides housing, training and employment initiatives to help the homeless off the streets. The Big Issue subsidiary «Making It» will employ ex-vendors in the development and marketing of commercial products.

The Big Issue is funded through advertising, business sponsorship and sales. All profits go back into helping homeless people.

The Big Issue cannot reply to any correspondence unless it is accompanied by an SAE. We are not responsible for unsolicited articles, photos or artwork received. The articles in «Street Lights* » are originals and do not necessarily represent The Big Issue's editorial philosophy, or the views of other homeless people.
}

* Note for the translator: «Street Lights» is the column for letters from the readers. 